\title{
Stage I Gastric Cancer AJCC v7
}

National Cancer Institute

\section{Source}

National Cancer Institute. Stage I Gastric Cancer A/CC v7. NCI Thesaurus. Code C89856.

Stage I includes: IA (T1, N0, M0); IB (T2, N0, M0); (T1, N1, M0). T1: Tumor invades

lamina propria, muscularis mucosae, or submucosa. T2: Tumor invades the muscularis

propria. N0: No regional lymph node metastasis. N1: Metastasis in 1-2 regional lymph nodes. M0: No distant metastasis. (AJCC 7th ed.) 